\title{
LIFT-the Italian Link for Time and Frequency
}

\author{
F. Levi, D. Calonico, A. Mura, M. Frittelli, \\ C.E. Calosso and M. Zucco \\ INRIM, Torino, Italy.

\section{Clivati and G. A. Costanzo.} \\ Politecnico di Torino and INRIM, Torino, Italy.
}

\section{R. Ambrosini.}

INAF - Istituto di Radioastronomia, Bologna, Italy.

\begin{abstract}
We report on the realization of the coherent optical link for time and frequency dissemination developed in Italy. The fiber backbone connects scientific laboratories that need accurate time and frequency measurements located in Torino, Milano, Bologna and Firenze. We briefly describe the technique, and report the status of installation and preliminary characterization.
\end{abstract}

\section{Keywords-Fiber link, Time and Frequency transfer}

\section{INTRODUCTION}

Optical fiber links are a key enabling technology for science and metrology. They are suitable for the remote comparison of very accurate clocks, such as Cs fountains or optical clocks [1-3] and their resolution exceeds the state-ofthe-art satellite techniques by several orders of magnitude. The coherent optical link allows to exploit the accuracy of an optical clock in few hundred seconds over hauls up to hundreds of $\mathrm{km}$, a performance otherwise impossible. This is an outstanding issue, as optical frequency standards are recommended by the BIPM for the secondary representation of the second in the International System (SI) of units, and are the most promising candidates for its future redefinition [4]. It is possible to achieve frequency dissemination (and comparisons) at the $10^{-16}$ level of stability $[2,3]$ and time dissemination at the $100 \mathrm{ps}$ level [5] over hauls of $1000 \mathrm{~km}$. This level of resolution needs for the compensation of the phase noise introduced by the fiber due to mechanical stresses and temperature variations, using a bidirectional Doppler cancellation technique [6].

The National Institute of Metrology (INRIM) realizes in Italy accurate $\mathrm{T} \& \mathrm{~F}$ signals and disseminates them to a variety of scientific laboratories and industries. Presently, INRIM maintains two Cs fountain primary frequency standards, with an accuracy at the $10^{-16}$ level [7], and is developing an optical clock based on $\mathrm{Yb}$ atoms [8]. To improve the quality of $\mathrm{T} \& \mathrm{~F}$ dissemination, INRIM started together with other major National Institutions, the LIFT project (the Italian Link for Frequency and Time, funded within Progetti Premiali 2012), to realize an optical link along a $642 \mathrm{~km}$ fiber that connects Torino to Milano, Bologna, and Firenze.

\author{
G. Galzerano, \\ CNR-IFN, Milano, Italy.
}

\author{
P. De Natale and D. Mazzotti \\ CNR-INO, Firenze and Sesto Fiorentino (FI), Italy.
}

\author{
N. Poli D. V. Sutyrin, and G. M. Tino \\ Università di Firenze, LENS and INFN, Sesto Fiorentino
}

(FI), Italy

The aim of the project is the distribution of the INRIM reference $T \& F$ signals to a variety of scientific laboratories via optical fiber, with a target relative instability of $10^{-14}$ at $1 \mathrm{~s}$ and $10^{-18}$ at one day measurement time. Those laboratories represent national centers of excellence in their fields; this facility will further improve their scientific capabilities, allowing them to better exploit existing experiments and to establish new ones in the future.

Present partners of the project are the National Institute of Nuclear Physics in Firenze (INFN), in collaboration with the European Laboratory for Non-linear Spectroscopy (LENS) and Department of Physics and Astronomy of University of Firenze; the National Institute of Astrophysics (INAF) in Medicina, near Bologna, and the National Council of Research (CNR) through the National Institute of Optics (INO) in Firenze and the Institute of Photonics and Nanotechnologies (IFN) in Milano.

Both INFN and CNR are involved in high resolution spectroscopic measurements, and will benefit from $T \& F$ dissemination via optical fiber. In particular, at LENS a $\mathrm{Sr}$ optical clock is under evaluation [9], and its frequency comparison to the frequency standards of INRIM could allow a number of new fundamental physics experiments. The INAF radiotelescope can be both an high demanding user of the external T\&F standards as well as a source for their close inter comparison with Pulsar-derived time scales or with the Celestial Reference Frame defined via Very Long Baseline Interferometry (VLBI). Closing the loop between sky observations and the new atomic frequency standards, even spread in different Italian locations, could lead to detect subtle systematic effects or in any case improve both sides of science.

$T \& F$ measurements with improved resolution are expected to be performed from 2014, in the second stage of the project, whilst in the first start-up stage the fiber backbone will be fully characterized and preliminary tests and checks will be completed. 


\section{COHERENT TIME AND FREQUENCY DISSEMINATION THROUGH OPTICAL FIBER}

A key point of the project is to use the same infrastructure to disseminate both time and frequency. When performing frequency dissemination, an ultrastable laser $(1542 \mathrm{~nm})$ is sent to the user, and its frequency is simultaneously measured in the two laboratories, with the aid of optical frequency combs. The frequency of the delivered laser is perturbed by environmental noise in the fiber. However, it is possible to compensate this noise with an optoacoustic actuator; this technique requires that the end laboratory redirects the incoming signal to the start laboratory, where the delivered signal is compared with the received one, and the added noise is cancelled in real time. For the noise cancellation to be effective, there must be a fully-optical backbone with no optical/electrical conversions, and the light has to travel exactly the same path in both directions. This is quite different from typical fiber transmission techniques, and poses several requirements on the optical instrumentation.

To disseminate time signals, a pseudorandom noise will be encoded on the optical carrier, similarly to the two way satellite time and frequency transfer: instead of cancelling the phase noise of the carrier itself, the measured transmission delay will be calibrated and stabilized, with a resolution of 100 ps.

The optical fiber used in our project is a $\sim 640 \mathrm{~km}$ dedicated fiber, with a total loss of $\sim 180 \mathrm{~dB}$, due to hauls and connectors losses. To adequately compensate for optical losses, we installed dedicated, fully bidirectional ErbiumDoped Fiber Amplifiers (EDFAs) along the path, that use the same active fiber in both directions (Figure 1).

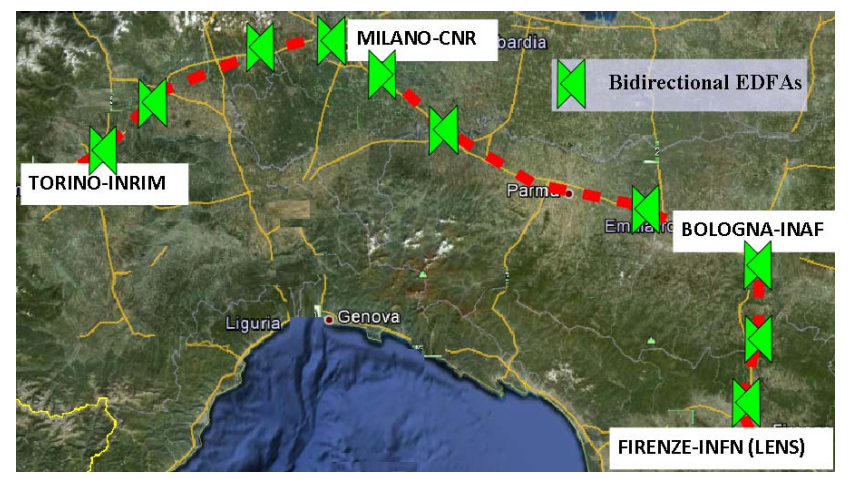

Fig. 1. The map shows the optical path and amplification sites.

\section{LINK IMPLEMENTATION AND CHARACTERIZATION}

One of the demanding steps of the link implementation is to compensate for the optical losses in such a bidirectional transmission scheme: the gain of our dedicated EDFAs could be set only with the whole link operating, as the gain and optical signal-to-noise ratio (OSNR) of each stage is affected by the others. Setting the gain below $\sim 22 \mathrm{~dB}$, we prevent oscillation lasing effects and OSNR degradation due to backscattering and Amplified Spontaneous Emission (ASE) [10]. Currently, 9 amplifiers have been installed, and optical filters have been used to keep the OSNR at an adequate level. In Figure 1, the optical path is shown with the location of the amplification sites; Table 1 reports for each station the distance from Torino and the optical losses of the haul. Measurements of the phase noise added by the fiber was performed during EDFA installation on several amplification sites, by reflecting the signal back towards INRIM. These measurements allowed us to check the noise contribution from each haul and the phase-compensation capabilities of the control loop.

Figure 2 shows the phase noise power spectral density of the back-reflected signal without the Doppler cancellation (free running fiber link). It represents the phase noise of the fiber up to three different intermediate stations. It is worth noting that the phase noise accumulated in the BolognaFirenze track $(100 \mathrm{~km})$ is comparable to the remaining part of the link $(450 \mathrm{~km})$ and exceeds the expected figure by several $\mathrm{dB}$. The reason for this excess of noise are under investigation with the fiber provider.

Figure 3 shows the phase noise power spectral density of the correction signal applied to the actuator when the compensation loop was activated on the full $642 \mathrm{~km}$ optical fiber link.

\begin{tabular}{|l|r|r|}
\hline \multicolumn{1}{|c|}{ Location } & \multicolumn{1}{c|}{ Length } & \multicolumn{1}{c|}{ Losses } \\
\hline Torino & $25 \mathrm{~km}$ & $9 \mathrm{~dB}$ \\
\hline Santhia & $67 \mathrm{~km}$ & $18 \mathrm{~dB}$ \\
\hline Novara & $77 \mathrm{~km}$ & $18 \mathrm{~dB}$ \\
\hline Lainate & $50 \mathrm{~km}$ & $15 \mathrm{~dB}$ \\
\hline Milano & $60 \mathrm{~km}$ & $18 \mathrm{~dB}$ \\
\hline Piacenza & $67 \mathrm{~km}$ & $16 \mathrm{~dB}$ \\
\hline Reggio Emilia & $94 \mathrm{~km}$ & $23 \mathrm{~dB}$ \\
\hline Bologna & $74 \mathrm{~km}$ & $19 \mathrm{~dB}$ \\
\hline Rioveggio & $38 \mathrm{~km}$ & $10 \mathrm{~dB}$ \\
\hline Firenze & $72 \mathrm{~km}$ & $18 \mathrm{~dB}$ \\
\hline LENS laboratories & $18 \mathrm{~km}$ & $7 \mathrm{~dB}$ \\
\hline
\end{tabular}

Table 1.Lenghts and losses of the various link hauls.

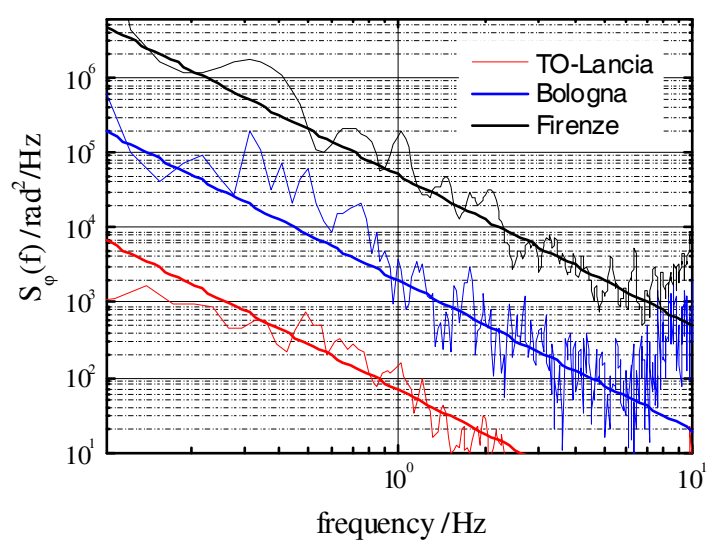

Fig. 2. Phase noise of several iber link hauls: Torino $(25 \mathrm{~km})$, Bologna $(+490$ $\mathrm{km})$ and Firenze $(+128 \mathrm{~km})$. It is evident that anomaluos noise is present in the track between Firenze and Bologna.

Based on these measurements we can estimate the ultimate achievable stability of the transferred signal at Milano, Bologna and Firenze. The value obtained for the Torino Firenze link in terms of Allan deviation in $1 \mathrm{~Hz}$ bandwidth is 
$3 \times 10^{-15}$. This estimate is based on the assumption that the phase noise cancellation bandwidth is limited by the time $(\sim 6$ s) it takes to the light to travel to the far fiber end and back [5].

Presently, we have completed and operated the link to Firenze but we have not yet extracted the signal for the intermediate users in Bologna and Milano. The laser transmitted from INRIM and delivered to LENS in Firenze is used as a reference to phase lock a $1542 \mathrm{~nm}$ diode laser. This laser is then frequency doubled and sent to a local Ti:Sa based optical frequency comb, to be compared with the local ultrastability laser at $698 \mathrm{~nm}$ used in the $\mathrm{Sr}$ clock experiment . In Figure 4 we report the frequency stability of the delivered laser measured against the local ultrastable laser in Firenze. There are also shown the stability of the free running fiber link (i.e. without noise compensation) and the stability of the 1542 $\mathrm{nm}$ laser at INRIM. The compensated link contribution is assessed to be $<1.5 \times 10^{-14}$ and does not degrade the stability performances of the delivered laser at $10 \mathrm{~s}$. The Allan deviation of the delivered laser was measured in Torino using a twin stabilized laser as reference.

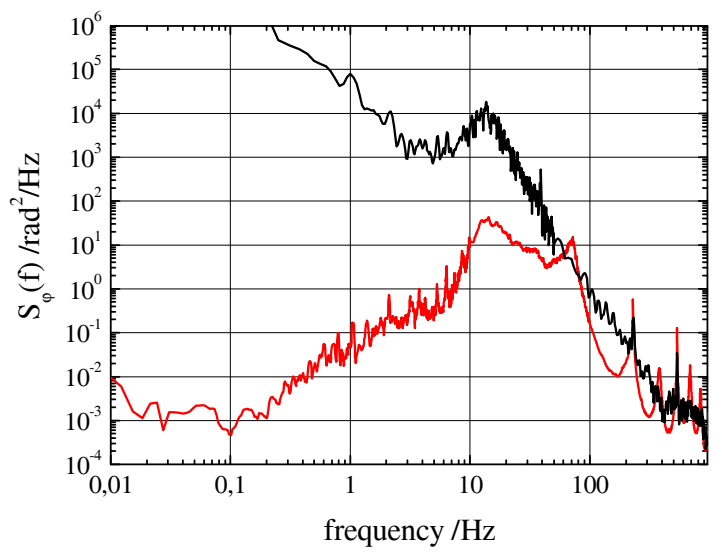

Fig. 3. Phase noise of the fiber link at Firenze $(642 \mathrm{~km})$, free running (black curve) and with Doppler compensation (red curve).

For an ultimate characterization, during next year, a second fiber link in an antiparallel configuration [2] is planned. This will allow us to determine the contribution of the non-compensated phase noise of the fiber itself, to detect possible sources of frequency inaccuracies, like cycle slips etc. and to state the ultimate stability performance of the optical link. Nevertheless, according to the preliminary characterization, the present optical link can already be used for frequency dissemination with high stability and accuracy.

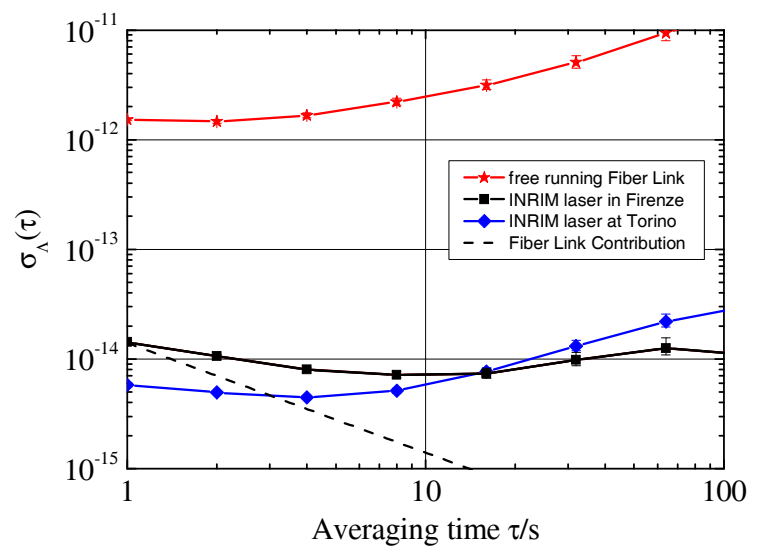

Fig. 4. Stability of the link: stablity of the free running link (red stars); stability of the INRIM $1542 \mathrm{~nm}$ laser measured at LENS vs the $698 \mathrm{~nm} \mathrm{Sr}$ clock laser in closed loop (black squares); stability of 1542nm laser measured at INRIM (blu diamonds) and the deduced contribution of the compensated link (dashed line). Note that the measurement of the laser stability at INRIM is not taken contemporarely to the one in Firenze, hence the fluctuation of the noise around $100 \mathrm{~s}$.

\section{CONCLUSIONS}

We report on the implementation and preliminary characterization of the $642 \mathrm{~km}$ fiber link connecting Torino to Milano, Bologna and Firenze within the LIFT project. At present, the fiber backbone has been implemented and preliminary characterization is being carried on. The amount of phase noise added by the optical fiber that cannot be compensated is not a limitation, and we estimated that frequency dissemination at the $10^{-16}$ level of stability in few minutes of averaging time can be obtained. Experiments related to time distribution will also be performed in the next months.

Fiber frequency dissemination represents a dramatic improvement with respect to satellite techniques, and enables frequency comparisons and calibrations at unprecedented levels of uncertainty, paving the way for a number of scientific experiments in the fields of high resolution spectroscopy, radio-astronomy, fundamental physics.

The development of this National network is of major interest also for high-tech industries, making available high quality $\mathrm{T} \& \mathrm{~F}$ signals in real time, without need of routine calibration. This infrastructure will be easily adapted to perform widespread dissemination towards a larger variety of users, with reduced costs. This secondary dissemination will use optical fibers as well, but instead of optical carrier it will distribute an amplitude modulated signal, much easier to be detected and used.

\section{ACKNOWLEDGMENTS}

The authors thank G. Santarelli, H. Schnatz, G. Grosche and P. E. Pottie for their help and advice.

University of Florence thanks PTB for providing the 698 nm laser used in the characterization of the link. 
University of Firenze, Politecnico of Torino and INRIM acknowledge the Italian Ministry of Education, University and Research (PRIN09-2009ZJJBLX) for funding the optical link.

INRIM acknowledges Compagnia di S. Paolo, the Italian Ministry of Education, University and Research (Progetti Premiali 2012), and the EMRP program (Project SIB02NEAT-FT) for funding. The EMRP is jointly funded by the EMRP participating countries within EURAMET and the European Union.

University of Firenze acknowledges Ente Cassa di Risparmio di Firenze for funding.

The authors acknowledges GARR for the technical help with the fiber.

\section{REFERENCES}

[1] C. W. Chou, D. B. Hume, J. C. J. Koelemeij, D. J. Wineland, and T. Rosenband, "Frequency Comparison of Two High-Accuracy Al+ Optical Clocks," Phys. Rev. Lett. 104, 070802 (2010).

[2] O. Lopez, A. Haboucha, F. Kefelian, H. Jiang, B. Chanteau, V. Roncin, C. Chardonnet, A. Amy-Klein, G. Santarelli, "Cascaded multiplexed optical link on a telecommunication network for frequency dissemination," Opt. Expr. 18, 16849-16857 (2010).

[3] K. Predehl, G. Grosche, S. M. F. Raupach, S. Droste, O. Terra, J. Alnis, Th. Legero,T. W. Hansch, Th. Udem, R. Holzwarth, H. Schnatz, "A 920-Kilometer Optical Fiber Link for Frequency Metrology at the 19th decimal place," Science 336, 441-444 (2012).

[4] Bureau International Des Poids et des Mesures, Consultative Committee for Time and Frequency (CCTF), Report of the 19th meeting (13-14 September 2012) to the International Committee for Weights and Measures, http://www.bipm.org/utils/common/pdf/CCTF19.pdf

[5] O. Lopez, A. Kanj, P. Pottie, D. Rovera, J. Achkar, C. Chardonnet, A. Amy-Klein, G. Santarelli "Simultaneous remote transfer of accurate timing and optical frequency over a public fiber network" Appl. Phys. B 110, 3-6 (2013).

[6] W. Williams, W. C. Swann, and N. R. Newbury, "High-stability transfer of an optical frequency over long fiber optic links," J. Opt. Soc. Am. B 25, 1284-1293 (2008).

[7] F. Levi, C. calosso, D. Calonico, L. Lorini, E. K. Bertacco, A. Godone, G. A. Costanzo, B. Mongino, S. Jefferts, T. P. Heavner, E. A. Donley "Cryogenic Fountain Development at NIST and INRIM: Preliminary Characterization", IEEE Trans. Ultrason. Ferroelectr. Freq. Contr. 57, 600-605, (2010).

[8] M. Pizzocaro, G. A. Costanzo, A. Godone, F. Levi, A. Mura, M. Zoppi, D. Calonico, "Realization of an Ultrastable $578 \mathrm{~nm}$ Laser for an $\mathrm{Yb}$ Lattice Clock", IEEE Trans. Ultrason. Ferroelectr. Freq. Contr. 59, 3, 426431 (2012).

[9] N. Poli, M. G. Tarallo, M. Schioppo, C. W. Oates and G. M. Tino "A simplified optical lattice clock", Appl. Phys. B 97, 27-33 (2009).

[10] L. Sliwczynski and J. Kolodziej, "Bidirectional Optical Amplification in Long-Distance Two-Way Fiber-Optic Time and Frequency Transfer Systems", IEEE Trans. Instrum. Meas. 62, 1, 2013. 